\title{
The Evaluation of Effectiveness for the Collaborative Combat of an Unmanned Aerial Vehicle Based on Grey Minimum Entropy
}

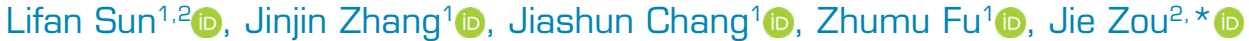

1. Henan University of Science and Technology - School of Information Engineering- Luoyang - China. 2.Science and Technology on Electro-optic Control Laboratory - Luoyang - China.

*Corresponding author: Chuba0920@163.com

\begin{abstract}
The main points to the evaluation of effectiveness for the collaborative combat of the unmanned aerial vehicle (UAV) lie within the construction of a reasonable indicator system and an accurate contribution model. As for point one, this article introduces a new method combining the Delphi consulting method and the principal component analysis method to avoid the underlying subjective and time-consuming defects of the existing methods. As for another point, a weighting method is adopted combining the subjective and objective parameters to minimize the errors caused by a single entity. Firstly, the modified grey relational degree analysis method is used to obtain the subjective weight, which can reduce the influence of the extreme values and outliers by enhancing the selection process of the reference sequence. Secondly, this paper adopts the weight of minimum entropy weight method to obtain the objective weight; it can avoid the information loss caused by the original method, which only determines the weight based on the frequency of each element present in the effective combination. At last, the principle of minimum relative entropy is adopted to obtain a more reasonable weight distribution coefficient. The simulation experiments established the rationality and effectiveness of the proposed method.
\end{abstract}

Keywords: Principal component analysis; Grey relational degree analysis; Minimum relative entropy.

\section{INTRODUCTION}

In recent time, with the quick development of artificial intelligence technology and electronic information technology in the military field, it is seen that the traditional mode of combat has gradually changed to become more intelligent and informational. As a new form of modern warfare, the unmanned aerial vehicle (UAV) cluster collaborative combat emerges with excellent survivability on battlefields along with mission completion capability. Through cooperative detection, cooperative tracking, cooperative attack, cooperative interaction, and a cooperative interception, multiple UAVs can effectively improve operational capabilities in complex environments and even complete operational tasks unmanned or with reduced participation.

Received: Feb. 6, 2021 | Accepted: Apr. 9, 2021

Peer Review History: Single Blind Peer Review.

Section Editor: Joana Ribeiro

This is an open access article distributed under the terms of the Creative Commons license. 
At present, the UAV cluster collaborative combat system is still in the developmental stage, and there are only a few research achievements regarding its evaluation in combat effectiveness. Since the actual combat environment is complex and unstable, the effectiveness in combat is affected by a variety of uncertain factors. However, a financial loss is created if a large number of physical tests are used to evaluate the effectiveness (Fan et al. 2018). Therefore, it is particularly important to evaluate the effectiveness of the multi-aircraft cooperative combat, whether from the perspective of promoting the integration of new combat forces with the joint combat system, improving the actual level of combat training of the troops, or improving the theoretical system of the weapon and the combat equipment (Jiang et al. 2020).

The effectiveness evaluation process of the UAV collaborative combat mainly consists of two parts, that is, the establishment of a reasonable indicator system and an accurate contribution model. While constructing the indicator system, the Delphi consulting method is commonly used from the existing literature to select the indicator. The Delphi consultation method was used to efficiently evaluate new models based on expert experience (Zhang and Xi 2021), which makes the entire evaluation process more authoritative and scientific. However, there are disadvantages regarding the factors of strong subjectivity and time consumption. To solve the subjective problem of the traditional Delphi consultation method, some studies suggested few solutions, which include improving the design content of the consultation form, statistical analysis methods, and the process of repeated consultation (Hanson et al. 2020; Wang et al. 2016). However, because of the timeconsuming defect of the Delphi method, there is no efficient method that can be adopted to improve it. Currently, the principal component analysis (PCA) method is widely used in the construction of various evaluation indicator systems as a method for mathematical dimensionality reduction. An earlier study adopted the PCA method to establish a comprehensive decision-making model concerning the threat of the artillery target, which helps in quickly processing data and make effective decisions (Wang et al. 2017).

Accurate contribution modeling on the basis of the established indicator system is the key to solve the problem of the effectiveness evaluation in the UAV collaborative combat system. The contribution is a measure of how much an indicator contributes to the whole system, and it is commonly referred to as weight. The methods used to evaluate the contribution of the system include subjective weighting, objective weighting, and combination weighting. Subjective weighting methods include the analytic hierarchy process, fuzzy matter-element analysis, grey relational analysis, and so on. In an earlier study by Qin et al. (2020), the analytic hierarchy process was used to determine the subjective weights in the effectiveness of evaluation in the anti-ship missile combat system, which effectively solves the problem of quantification of the qualitative indicators by constructing a relative importance matrix. However, the disadvantage of strong subjectivity still exists. In an earlier study by Li et al. (2020), gray correlation was used to construct an evaluation model for the water conservancy project risk analysis, but there are outliers which affect greatly while selecting the optimal sequence.

The objective weighting methods mainly include the method of the coefficient of variation, the maximum deviation method, and the entropy weighting method, respectively. The coefficient of variation method was adopted to determine the objective weight, which can eliminate the effect of different dimensions and does not need to consider the normalization of the indicator value (Li et al. 2020). However, it often leads to uncertainty and incorrect weight estimation once the outliers appear in the data. In an earlier study (Tian et al. 2004), the maximum deviation method was adopted, which is based on the difference in the indicator value. However, a greater difference between the values of an indicator does not mean that the indicator is more important, which results in a significant difference between the weighting results and the actual importance of the indicator. The entropy weight method was adopted, which can avoid the problem of bias caused by the subjective weighting method (Luo et al. 2019; Tong et al. 2011). However, there is a defect that may ignore the importance of indicators themselves. The principle of minimum entropy analysis was applied to obtain the objective weight, and the mutual influence between the indicators is considered, which greatly improves the objectivity of the selection of an indicator (Shan et al. 2014). The same study also applied the principle of minimum entropy analysis to obtain the objective weight, which takes into account the interaction between the indicators and greatly improves the objectivity of the indicator selection. However, it only determines the weights based on the frequency of each indicator in the effective combination, ignoring the difference in the relative entropy among different combinations. 
In view of these problems, this paper proposes the following modified methods: as for how to construct a reasonable indicator system, to deal with the time-consuming and subjective shortcomings of the original method, this paper modifies it by combining the PCA method and the Delphi consulting method, respectively. With that, the indicator can be selected more objectively and processed quickly; thus, the process of decision-making can be more effective when the quality of the indicator increases. In order to construct an accurate contribution model, this paper presents a joint effectiveness evaluation model combining the subjective and objective methods and uses the principle of minimum relative entropy to calculate a more reasonable weight distribution coefficient. In this case, for constructing a subjective contribution model, the minimum entropy is used to improve the selection process of the reference sequence in the gray correlation analysis method, which can reduce or even eliminate the influence of extreme values and outliers. Also, this paper adopts an improved minimum entropy weight method to construct an objective evaluation model. Besides, this paper formulates the rules for deciding and selecting an effective combination of indicators links. It links the relative entropy value with the value of weight data to avoid the problem of information loss in the traditional method. Through the example of analysis, it is proved that the evaluation methods proposed in this paper are reasonable and effective.

\section{CONSTRUCTION OF THE INDICATOR SYSTEM}

In this paper, the expert consultation method and the PCA method are combined to select every indicator in the evaluation system. That is to say, the evaluation value of each indicator is obtained through a series of questionnaire recovery, the PCA method is used to screen out the indicators that have a greater impact on the evaluation system. The specific steps are given as follows:

1. After defining the object of evaluation, an initial set of indicators are formulated and evaluation experts are selected. It is necessary for all the selected experts to be experienced in the research field, to avoid subjective arbitrariness of the statistical results caused by different levels of expertise.

2. The preliminary set of indicators is distributed to the experts in the form of a consultation form, and each expert is required to score the importance of each indicator concerning its upper-level indicator. For quantifiable indicators, the score range is taken as a percentage. For indicators that require qualitative analysis, the degree of importance is divided into five levels: very important, relatively important, generally important, less important, and unimportant. The percentage score of each grade is shown in Table 1.

Table 1. Classification of importance levels.

\begin{tabular}{cccccc}
\hline Level & Unimportant & Less important & Generally important & Relatively important & Very important \\
\hline Scores & 50 & 60 & 70 & 80 & 90 \\
\hline
\end{tabular}

3. The value of each indicator is evaluated and the data is normalized, then the PCA is used to obtain the variance in the contribution degree of each indicator. Here, the PCA is a feature extraction algorithm having practical significance. The original multiple indicators can be transformed into a new set of independent indicators by using the PCA, and the selected principal components must reflect the information of the original indicator group as much as possible. The amount of information contained can be measured by the variance contribution. The several current indicators, whose cumulative variance contribution reaches up to $100 \%$, can be considered to contain all the information of the original indicator group. From a larger to smaller variance contribution, it is called the first principal component and the second principal component, and so on.

4. The variance contribution of each indicator is accumulated to obtain the cumulative variance contribution. In actual research, when the cumulative variance contribution reaches up to $85 \%$, it can be concluded that the main components have been screened out. The whole process of constructing an evaluation indicator system is shown in Fig. 1. 


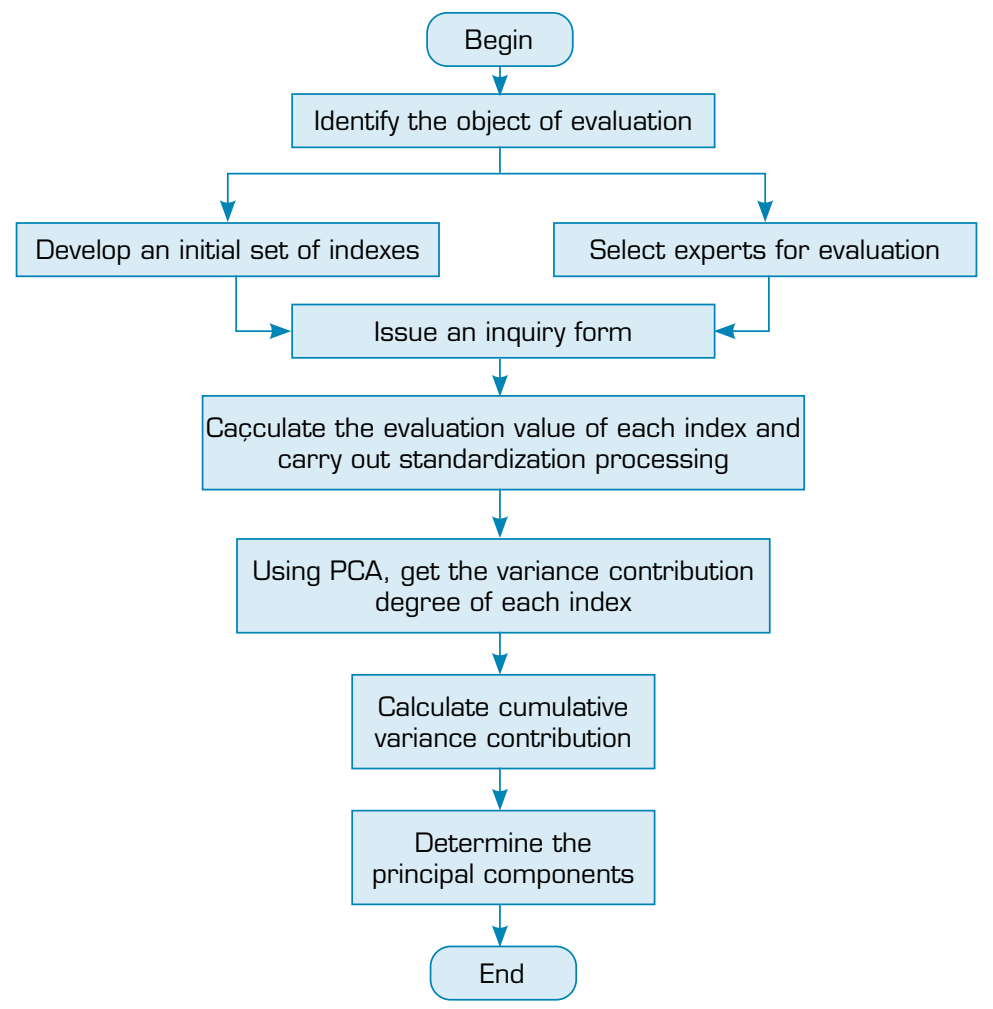

Figure 1. Flow chart of expert consultation method combined with PCA.

In this work, the expert PCA method is considered to develop the evaluation indicator system, which not only greatly reduces the workload of the indicator selection, but also reduces the subjective arbitrariness of the evaluation process.

\section{ESTABLISHMENT OF THE CONTRIBUTION MODEL}

As for establishing the contribution model, the evaluation method of the contribution of each indicator can be roughly divided into the subjective and the objective weighting methods. The subjective weighting method is mainly determined by experts and shows a certain degree of authority and reliability; thus, there will be no serious deviation in the weights from the factual values. However, the dissimilarities between experience and knowledge would inevitably cause certain subjective arbitrariness. The objective weighting method calculates only based on the data, avoiding the influence of subjective factors; however, it could lead to a considerable difference between the final weighting result and the actual importance of the indicator. Therefore, this paper adopts a combination of the subjective and objective weighting methods to achieve the purpose of complementing the advantages of the two. Besides, the principle of minimum relative entropy is adopted to obtain a more reasonable distribution coefficient of the weight.

\section{Subjective evaluation model based on the grey relational degree}

Along with their independence, the indicators present in the UAV collaborative combat effectiveness evaluation system also affect each other, but the degree of such effect is unknown. Therefore, while determining the weight of each indicator, it is not only necessary to calculate the importance of a single indicator to the upper layer, but also to consider the degree of influence on the other indicators of the same layer. The degree of influence can be measured by the size of the gray correlation coefficient, so for the effectiveness evaluation system having relative influence among indicators, the gray correlation degree analysis method can be used to determine the subjective weight. 
Here, selecting a reference sequence is a key step in the gray correlation analysis method. While determining the elements in the reference sequence, the traditional method generally chooses the maximum value of the positive indicator and the minimum of the negative indicator, or the average value. Although the method is simple, information loss could occur easily by just overemphasizing extreme values and ignoring the other values of evaluation. From the perspective of the whole system, the selected optimal element should reflect the information of the values of evaluation from this group; that is, it should be very close to each value of evaluation in this group. Therefore, this article uses a modified grey relational analysis method to construct the evaluation model to obtain the subjective weight of each indicator. The specific steps are as follows:

1. Determine the matrix that represents the importance of an indicator. Assuming that there are $n$ indicators in the same layer and $m$ experts are arranged to rate their importance, a matrix having $m$ rows and $n$ columns could be obtained (Eq. 1).

$$
\mathrm{X}=x_{m n}=\left[\begin{array}{cccc}
x_{11} & x_{12} & \cdots & x_{1 n} \\
x_{21} & x_{22} & \cdots & x_{2 n} \\
\vdots & \vdots & \ddots & \vdots \\
x_{m 1} & x_{m 2} & \cdots & x_{m n}
\end{array}\right]
$$

where $x_{0_{j}}$ represents the optimal value of the indicator $j, x_{i j}$ represents the evaluation value of the indicator $j$ given by the expert $i$. Calculate the optimal value of $n$ indicators, $x_{0}=\left(x_{01}, x_{02}, \ldots x_{0 n},\right), x_{0}$ is the reference sequence.

2. Determine the reference sequence. The elements present in the reference sequence corresponding to the same indicator are required to be close to each evaluation value of the indicator. This proximity can be measured by the relative entropy value, so the value that can minimize the sum of the relative entropy of itself and other evaluation values is the reference value of this group. The relative entropy between the reference value and any evaluation value is calculated as follows (Eq. 2):

$$
\min Q\left(x_{0 j}, x_{i j}\right)=\sum_{j=1}^{n} x_{0 j} \ln \left(\frac{x_{0 j}}{x_{i j}}\right)
$$

where $x_{0_{j}}$ represents the optimal value of the indicator $j, x_{i j}$ represents the evaluation value of the indicator $j$ given by an expert $i$. Calculate the optimal value of $n$ indicators, $x_{0}=\left(x_{01}, x_{02}, \ldots x_{0 n},\right), x_{0}$ is the reference sequence.

3. Calculate the absolute difference between the evaluation value and the corresponding reference value of the indicator $j$, individually (Eq. 3).

$$
\Delta=\left|x_{0 j}-x_{i j}\right|
$$

4. Calculation of the correlation coefficient (Eq. 4). The correlation coefficient of $A$ and $B$ concerning the indicator $j$ is:

$$
\varepsilon\left(x_{0 j}, x_{i j}\right)=\frac{\min _{i} \min _{j}\left|x_{0 j}-x_{i j}\right|+\max _{i} \max _{j}\left|x_{0 j}-x_{i j}\right|}{\left|x_{0 j}-x_{i j}\right|+\rho \max _{i} \max _{j}\left|x_{0 j}-x_{i j}\right|}
$$

where $\rho$ is the resolution reference, $0 \leq \rho \leq 1$, the smaller the value of $A$, the bigger the difference between the correlation coefficients. Usually, $\rho$ is 0.5 .

5. Calculation of relevance. The average value of the correlation coefficient of each indicator is the gray correlation degree.

6. Determination of weight. The weight of each indicator can be obtained by normalizing the correlation degree of every indicator. The whole process of constructing the subjective evaluation model is shown in Fig. 2. 


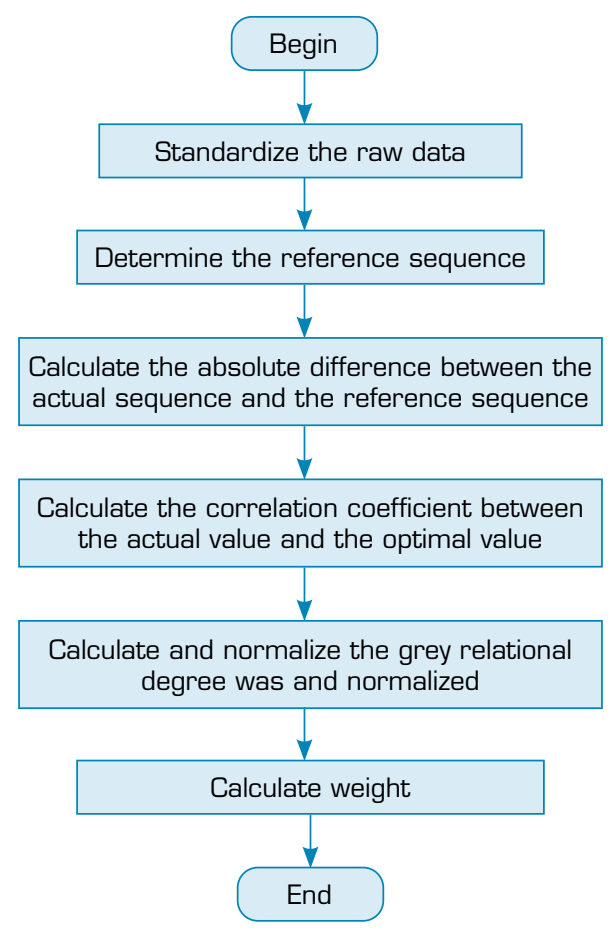

Figure 2. Flow chart of the modified grey relational degree analysis method.

This paper constructs an evaluation model based on the modified gray correlation analysis method and uses the principle of minimum relative entropy to determine the elements present in the reference sequence. This method comprehensively considers the influence of each evaluation value of the indicator and greatly reduces the effect of the extreme values and outliers on the selected optimal data, thereby obtaining a more reasonable and subjective weight.

\section{Objective evaluation model based on minimum entropy}

In the evaluation system, the indicators influence each other, and the degree of influence can be objectively determined by considering the relative entropy. The relative entropy, also known as mutual entropy, and cross-entropy, etc., can be used to measure the degree of mutual influence between the two indicators. Let $A(x)$ and $B(x)$ be the two probability distributions of the value of $x$, then the relative entropy of $A$ to $B$ is (Eq. 5):

$$
\mathrm{D}(\mathrm{A} \| \mathrm{B})=\sum_{i=1}^{n} A(x) \ln \frac{A(x)}{B(x)}
$$

The smaller the relative entropy of the indicator $A$ to $B$ is, the bigger will be the degree of influence the indicator $A$ has on $B$. Therefore, if the entropy of indicator $A$ compared to the other indicators in the evaluation system is very small, that is, the degree of influence is larger, the importance of the indicator $A$ in the entire system is higher; thus, more weight should be provided.

In the traditional method, the steps of using the principle of minimum entropy analysis to determine weight are as follows: first, combine $n$ indicators within the same layer to form different combinations, and calculate the relative entropy of each combination. Secondly, according to a certain judgment rule, the combination having the smallest relative entropy of the first few items is selected as the effective combination. Finally, calculate the frequency of each indicator present in the effective combination, and the normalized value is taken as the weight of each indicator.

However, the method mentioned only counts the frequency of each indicator in the effective combination to determine the weight, but ignores the difference in the relative entropy of each combination. For example, the relative entropy of the indicator $A$ to the indicator $B$ is 0.1 , and that of indicator $C$ to indicator $B$ is 0.2 . Even though both of these combinations are selected as effective combinations, their relative entropy values are different; thus, it is not possible to assume that they have the same weight 
just relying on the same frequency of occurrence of indicator $A$ and the indicator $C$. Also, the two combinations do not reflect the degree of influence of the indicator $B$ on other indicators, and hence the weight of the indicator $B$ cannot be obtained accordingly. Therefore, this paper adopts the modified minimum entropy weight method to construct the evaluation model, to obtain the objective weight of each indicator. The specific steps are as follows:

1. Combine two indicators of the same layer to form combinations, calculate the relative entropy of each combination, and sort them from smaller to larger combinations.

2. Determine the effective combination. Since the relative entropy of each combination only represents the degree of influence shown by the first indicator on the second one, we can consider this combination to be an effective combination of the first indicator. When determining an effective combination, the following guidelines are followed:

- All indicators must be included.

- Suppose the entropy difference between the effective combination with the maximum entropy and the ineffective combination with the minimum entropy is $p$, and the range of the value of entropy in the determined effective combination is $q$, so it is essential for the condition $\mathrm{p} \geq 0.1 q$.

3. Count all the effective combinations of each indicator, calculate the sum of their relative entropy values and take the reciprocal; the normalized value obtained is the weight of each indicator. The whole process of constructing an objective evaluation model is shown in Fig. 3.

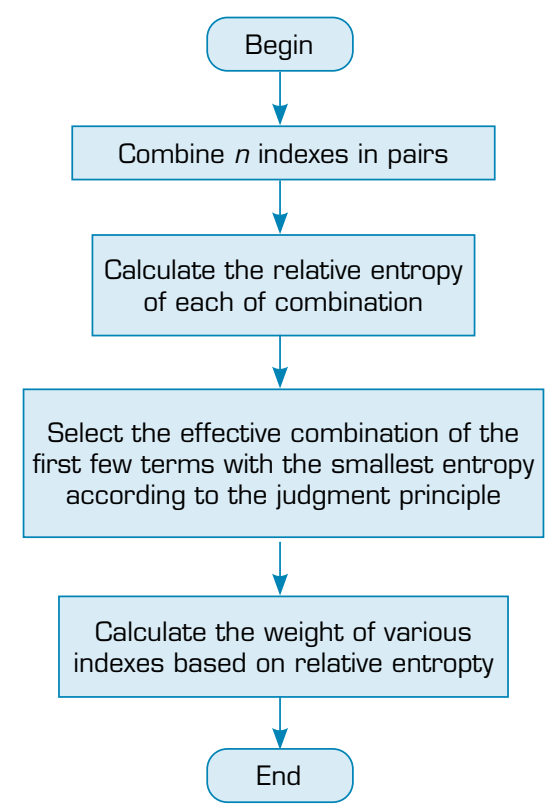

Figure 3. Flow chart of determining the weight by the minimum entropy weight method.

This article develops an objective evaluation model based on the modified minimum entropy method and employs relative entropy to determine the weight, which not only considers the mutual influence between the indicators but also weakens the influence of human factors on the weight determination result. Also, the relative entropy value and the value of the weight data are linked to avoiding information loss caused by determining the weight purely based on the frequency of each element in the effective combination.

\section{Joint effectiveness evaluation model}

The combination weighting method, which adds to the subjective weighting method and the objective weighting method according to a certain integrated algorithm, can combine the advantages of the two to make the final weight more reasonable (Zhang and $\mathrm{Xu}$ 2017). Here, the focus is on the distribution of weights; the combined weights are required to be closed to those obtained by the subjective and objective methods. Thus, a mathematical model based on the idea of minimum relative entropy can be established (Eq. 6). 


$$
\left\{\begin{array}{l}
\min Q(a, b)=\sum_{j=1}^{n} w_{j} \ln \left(\frac{w_{j}}{p_{j}}\right)+\sum_{j=1}^{n} w_{j} \ln \left(\frac{w_{j}}{q_{j}}\right) \\
a+b=1, w_{j}=a^{*} p_{j}+b^{*} q_{j}
\end{array}\right.
$$

where $p$ and $q$ are the weights obtained by the subjective weighting method and the objective weighting method, their weight distribution coefficients are $a$ and $b$, and $w$ is the combination of the two weights.

Therefore, this paper builds a mathematical model based on the original ideal of the minimum relative entropy to determine the distribution ratio of the subjective and objective weights, which balances the influence of the subjective and objective factors and enhances the weight distribution coefficient, and makes it more reasonable.

\section{INSTANCE VERIFICATION}

According to the different tasks involved, the entire multi-aircraft cooperative combat system includes the command center, reconnaissance aircraft, fighter aircraft, bombing aircraft, and other members, which can undertake the task of attacking ground targets or air targets. The structure diagram of the multi-aircraft combat system is shown in Fig. 4.

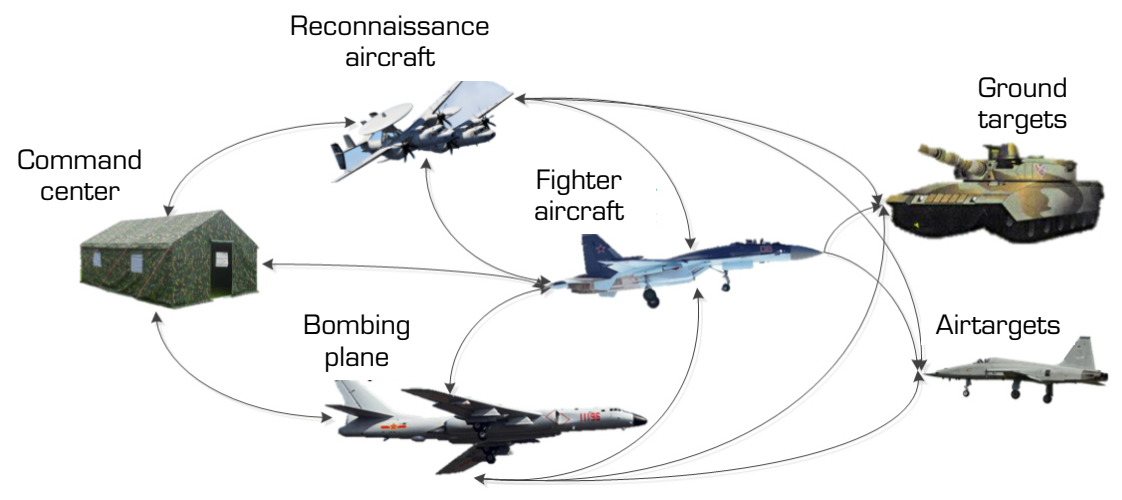

Figure 4. The combat architecture diagram of several fighter aircraft.

Here, the command center is responsible for the task of commanding and decision-making for the entire combat formation. Firstly, the operational conditions are judged by receiving feedback information from the reconnaissance aircraft. Secondly, the combat formation is adjusted according to the combat environment and the characteristics of the combat targets. Finally, judge the war situation and make real-time decisions based on the battlefield information (Wang et al. 2013). The tasks require the command center to have excellent decision-making ability (Wang et al. 2013). Among the members of the fighter formations, reconnaissance aircraft are mainly used to conduct reconnaissance and obtain intelligence from the air, fighter aircraft are mainly used to intercept enemy planes or guided missiles and, thus, gain air-superiority, while the bombing aircraft mainly undertake the task of attacking enemy artillery (Diao et al. 2014). Furthermore, the fighter formation members also need a real-time exchange of information, that is, cooperative exploration and communication capabilities are required. The established combat mission can be completed is the primary criterion for measuring the effectiveness of a formation coordinated combat, and the ability to strike the enemy is a key factor in determining the success or failure of a war, which is of vital importance to the effectiveness of the combat in the entire system. Therefore, collaborative exploration ability, command and decision-making ability, collaborative communication ability, and a collaborative attacking ability are taken as the key factors affecting the effectiveness evaluation of the multi-aircraft collaborative operations in this paper and are studied.

After the first round of the expert consultation method, the preliminary set of indicators in the UAV cooperative combat effectiveness evaluation system can be obtained, including the four subsystems and the indicator sets of the cooperative exploration ability, cooperative communication ability, cooperative attack ability, command, and decision-making ability, respectively. 
Then, the PCA is applied to screen all the indicators from the layer of indicators. The following content takes the collaborative attack capability, and its lower-layer indicator is set as an example for specific analysis.

Firstly, as per the method of expert consultation, 20 experts rate the quantitative analysis indicators of the fire control calculation ability, firepower striking ability, battlefield survivability, weapon damage ability, and equipment supply ability. As for the indicators that require qualitative analysis, experts need to state the level of importance. Also, they need to perform quantification processing according to the importance level division provided in Table 1, and the distribution diagram of the evaluation value of each indicator can be obtained as shown in Fig. 5. Finally, using the PCA to perform data dimensionality reduction processing, it is possible to obtain the degree of variance contribution of each indicator to the upper layer in Table 2 and the score coefficient table of each component in Table 3.

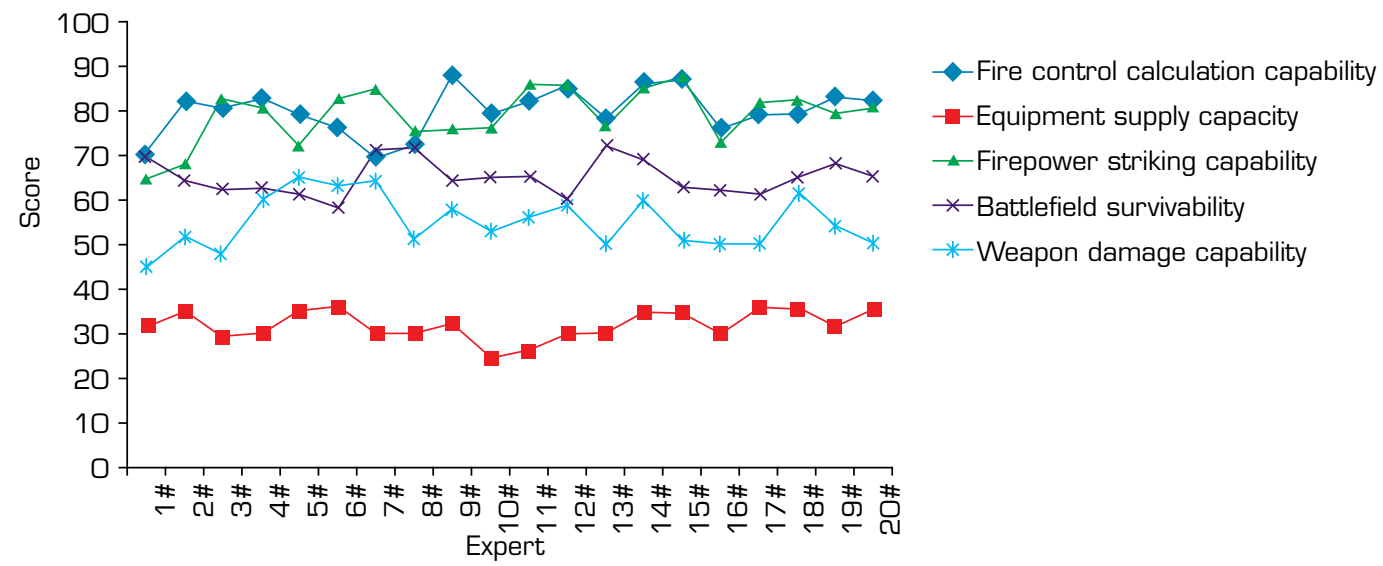

Figure 5. Expert evaluation value of each indicator.

Table 2. Total variance of each interpretation of the indicator.

\begin{tabular}{|c|c|c|c|c|c|c|}
\hline \multirow[b]{2}{*}{ Component } & \multicolumn{3}{|c|}{ Starting eigenvalue } & \multicolumn{3}{|c|}{ Extract the sum of squares and load } \\
\hline & Sum & Variance [\%] & $\begin{array}{l}\text { Accumulation } \\
\text { of variance [\%] }\end{array}$ & Sum & Variance [\%] & $\begin{array}{l}\text { Accumulation } \\
\text { of variance [\%] }\end{array}$ \\
\hline 1 & 1.387 & 27.744 & 27.744 & 1.387 & 27.744 & 27.744 \\
\hline 2 & 1.185 & 23.700 & 51.443 & 1.185 & 23.700 & 51.443 \\
\hline 3 & 1.113 & 22.268 & 73.711 & 1.113 & 22.268 & 73.711 \\
\hline 4 & 1.001 & 20.012 & 93.723 & 1.001 & 20.012 & 93.723 \\
\hline 5 & 0.314 & 6.277 & 100.000 & & & \\
\hline
\end{tabular}

Table 3. Score coefficient for each component.

\begin{tabular}{ccccc}
\hline \multirow{2}{*}{ Indicator } & \multicolumn{4}{c}{ Component } \\
\cline { 2 - 5 } & $\mathbf{1}$ & $\mathbf{2}$ & $\mathbf{3}$ & $\mathbf{4}$ \\
\hline Fire control calculation ability & 0.671 & 0.044 & -0.122 & 0.026 \\
\hline Equipment supply ability & 0.274 & -0.513 & 0.128 & 0.648 \\
\hline Firepower striking ability & 0.304 & 0.683 & 0.251 & 0.122 \\
\hline Battlefield survivability & -0.296 & 0.335 & -0.256 & 0.745 \\
\hline Weapon damage ability & -0.123 & -0.017 & 0.859 & 0.094 \\
\hline
\end{tabular}


When constructing the indicator system, the principles that must be followed while using the PCA have been provided. The cumulative variance in the contribution of the selected principal components lies in between 80 and $85 \%$, and the characteristic value of the selected principal components must be greater than 1 . According to the variance contribution degree of each indicator provided in Table 2, it can be seen that the degree in cumulative variance contribution of the first four indicators meets the requirements; thus, the number of indicators selected is four. Also, after combining the scoring coefficients of each component present in Table 3, it can be classified accordingly with the fire control calculation capability as the first principal component, the firepower striking ability as the second one, the weapon damage capability and the battlefield survivability as the third and the fourth one, respectively. Therefore, the lower-level indicator set of the cooperative attack capability includes the fire control calculation capability, firepower striking capability, battlefield survivability, and the weapon damage capability.

In the same way, the lower-level indicator set for the collaborative exploration capabilities includes the target positioning capability, target tracking capability, and anti-jamming capability. The lower-level indicator set for the collaborative interaction capability includes the information receiving capability, information processing capability, information sharing capability, and information fusion capability. The lower indicator set of the command and decision-making ability is the situational awareness, main decisionmaking, auxiliary decision-making abilities, and decision response time. The whole indicator system of the UAV collaborative combat effectiveness is shown in Fig. 6.

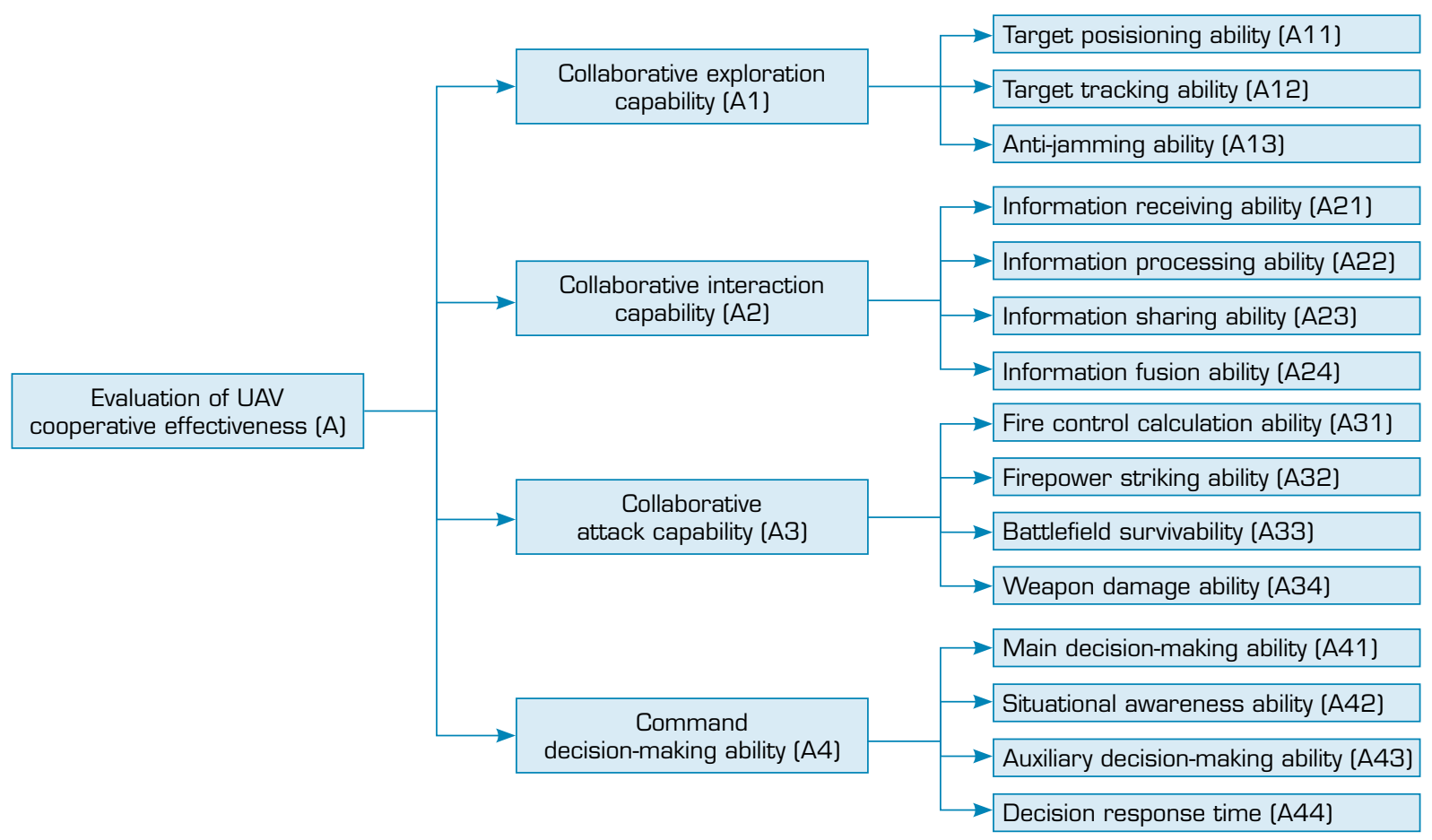

Figure 6. Evaluation indicator structure of UAV collaborative operation effectiveness.

The evaluation indicator system of the UAV collaborative combat effectiveness includes three levels, which can be named as the target level, the criteria level, and the indicator level. Among them, the criteria level has four indicators, and the indicator level consists of 15 indicators. The modified grey relational degree analysis method can be used to obtain the subjective weight of each indicator in the criterion layer and the indicator layer, as shown in Table 4.

The product of the weight of each indicator in the third layer and its corresponding indicator in the upper layer is the weight of each indicator relative to the whole system of evaluation. According to Eq. 6, the combined weight of each indicator can be obtained relative to the entire system. For every indicator in the indicator layer, the weights obtained by the three weighting methods are sorted out, respectively. 
Table 4. Subjective weight of each indicator.

\begin{tabular}{|c|c|c|c|c|}
\hline Target level & Criterion layer & Weight & Indicator layer & Weight \\
\hline \multirow{15}{*}{$\begin{array}{l}\text { Evaluation } \\
\text { of UAV } \\
\text { cooperative } \\
\text { effectiveness }\end{array}$} & \multirow{3}{*}{$\begin{array}{c}\text { Collaborative exploration } \\
\text { capability }\end{array}$} & \multirow{3}{*}{0.276} & Target positioning ability & 0.304 \\
\hline & & & Target tracking ability & 0.412 \\
\hline & & & Anti-jamming ability & 0.284 \\
\hline & \multirow{4}{*}{$\begin{array}{c}\text { Collaborative interaction } \\
\text { capability }\end{array}$} & \multirow{4}{*}{0.257} & Information receiving ability & 0.261 \\
\hline & & & Information processing ability & 0.200 \\
\hline & & & Information sharing ability & 0.292 \\
\hline & & & Information fusion ability & 0.247 \\
\hline & \multirow{4}{*}{$\begin{array}{c}\text { Collaborative attack } \\
\text { capability }\end{array}$} & \multirow{4}{*}{0.283} & Fire control calculation ability & 0.185 \\
\hline & & & Firepower striking ability & 0.277 \\
\hline & & & Battlefield survivability & 0.297 \\
\hline & & & Weapon damage ability & 0.241 \\
\hline & \multirow{4}{*}{$\begin{array}{l}\text { Command decision-making } \\
\text { capability }\end{array}$} & \multirow{4}{*}{0.183} & Main decision-making ability & 0.265 \\
\hline & & & Situational awareness ability & 0.215 \\
\hline & & & Auxiliary decision-making ability & 0.215 \\
\hline & & & Decision response time & 0.304 \\
\hline
\end{tabular}

The modified minimum entropy weight method is adopted to calculate the objective weight, and the objective weights of each indicator are obtained from the criterion layer and the indicator layer, as shown in Table 5.

Table 5. Objective weight of each indicator.

\begin{tabular}{|c|c|c|c|c|}
\hline Target level & Criterion layer & Weight & Indicator layer & Weight \\
\hline \multirow{15}{*}{$\begin{array}{l}\text { Evaluation } \\
\text { of UAV } \\
\text { cooperative } \\
\text { effectiveness }\end{array}$} & \multirow{3}{*}{$\begin{array}{c}\text { Collaborative exploration } \\
\text { capability }\end{array}$} & \multirow{3}{*}{0.322} & Target positioning ability & 0.278 \\
\hline & & & Target tracking ability & 0.453 \\
\hline & & & Anti-jamming ability & 0.269 \\
\hline & \multirow{4}{*}{$\begin{array}{c}\text { Collaborative interaction } \\
\text { capability }\end{array}$} & \multirow{4}{*}{0.255} & Information receiving ability & 0.211 \\
\hline & & & Information processing ability & 0.174 \\
\hline & & & Information sharing ability & 0.305 \\
\hline & & & Information fusion ability & 0.311 \\
\hline & \multirow{4}{*}{$\begin{array}{c}\text { Collaborative attack } \\
\text { capability }\end{array}$} & \multirow{4}{*}{0.255} & Fire control calculation ability & 0.221 \\
\hline & & & Firepower striking ability & 0.327 \\
\hline & & & Battlefield survivability & 0.302 \\
\hline & & & Weapon damage ability & 0.150 \\
\hline & \multirow{4}{*}{$\begin{array}{l}\text { Command decision-making } \\
\text { capability }\end{array}$} & \multirow{4}{*}{0.168} & Main decision-making ability & 0.202 \\
\hline & & & Situational awareness ability & 0.410 \\
\hline & & & Auxiliary decision-making ability & 0.198 \\
\hline & & & Decision response time & 0.190 \\
\hline
\end{tabular}


It can be seen from Table 6 that the subjective and objective weights of most of the indicators are the same. For such an indicator, the weight can be considered to be consistent with the actual importance of the indicator. However, the weights of A22, A32 and A42, obtained by the two methods, are relatively different. This is due to the errors caused by the inherent drawbacks of the subjective and objective weighting methods. However, as can be seen from the ranking of the combined weights, this error is greatly reduced, and the two weighting methods achieve the purpose of complementary advantages. Therefore, the actual effect of the joint effectiveness evaluation model constructed in this article is better than that of a single method, which verifies the effectiveness of the evaluation model. To prove the rationality of the model constructed in this paper, the overall effectiveness of the three formation schemes is evaluated as follows. The actual parameters of each indicator are shown in Fig. 7, and the evaluation results of all the schemes are shown in Fig. 8.

Table 6. Indicator weights calculated by different methods are sorted.

\begin{tabular}{|c|c|c|c|c|c|c|c|c|c|c|c|c|c|c|c|}
\hline & A11 & A12 & A13 & A21 & A22 & A23 & A24 & A31 & АЗ2 & АЗ3 & A34 & A41 & A42 & A43 & A44 \\
\hline $\begin{array}{l}\text { Subjective } \\
\text { weight }\end{array}$ & 1 & 2 & 5 & 8 & 12 & 6 & 9 & 11 & 4 & 3 & 7 & 13 & 15 & 14 & 10 \\
\hline $\begin{array}{l}\text { Objective } \\
\text { weight }\end{array}$ & 1 & 2 & 3 & 9 & 4 & 7 & 12 & 10 & 11 & 6 & 5 & 13 & 8 & 14 & 15 \\
\hline $\begin{array}{l}\text { Combined } \\
\text { weight }\end{array}$ & 1 & 2 & 3 & 8 & 7 & 5 & 12 & 11 & 9 & 4 & 6 & 14 & 10 & 15 & 13 \\
\hline
\end{tabular}

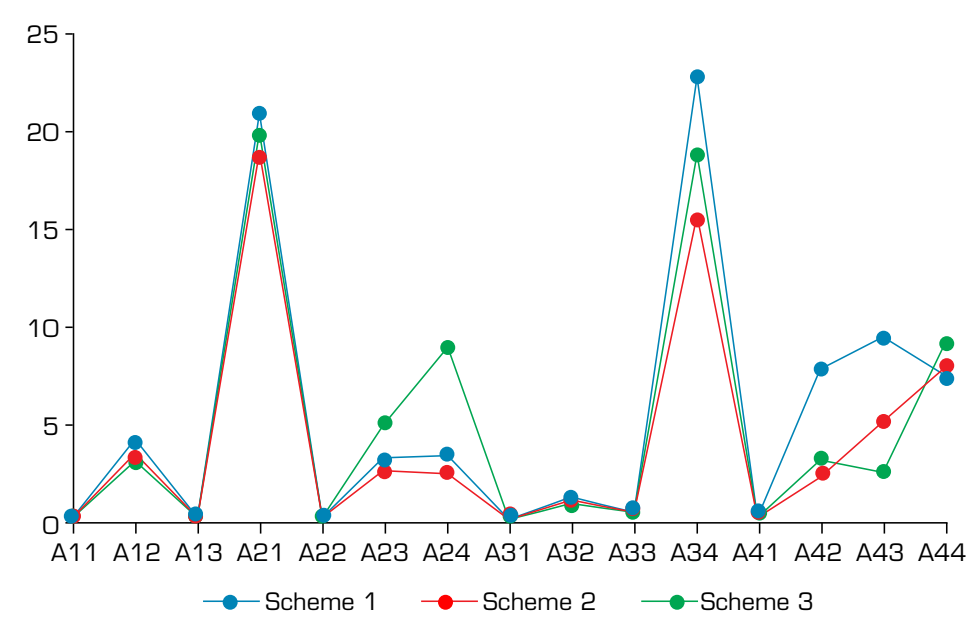

Figure 7. Parameters of each indicator under different formation schemes.

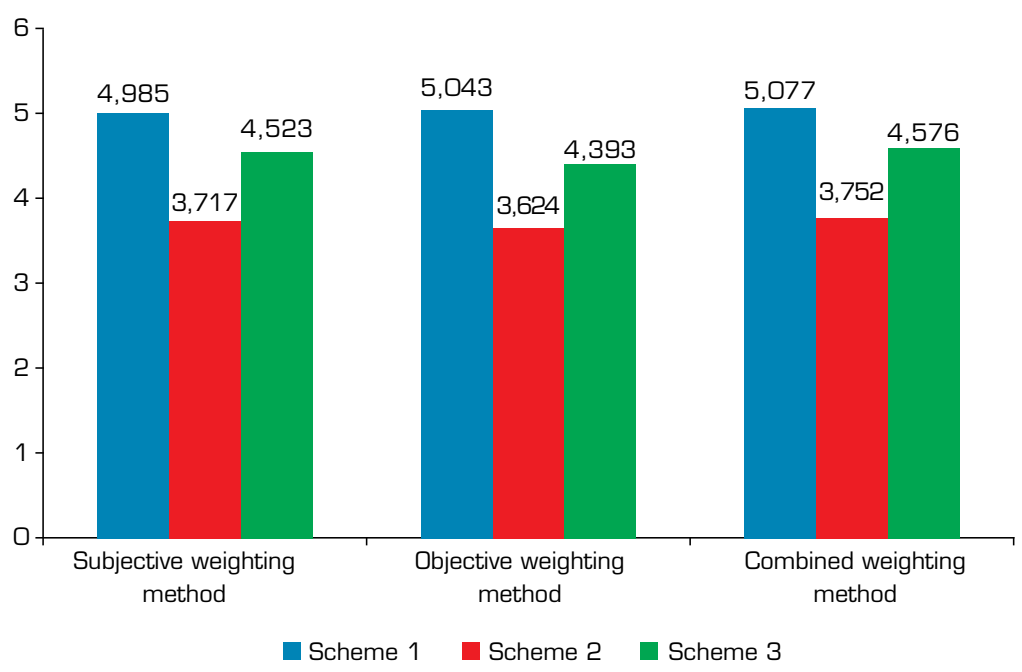

Figure 8. The effectiveness evaluation results of the three schemes under different weighting methods. 
It can be seen from Fig. 7 that the three different kinds of weighting methods are used to evaluate the combat effectiveness of the three different schemes of formation, and the results are: scheme $1>$ scheme $2>$ scheme 3 . This shows that the weights determined by the proposed method are consistent with the actual importance of the indicators, which verifies the rationality and feasibility of the evaluation model constructed in this paper.

\section{CONCLUSION}

In this paper, through the research on the effectiveness evaluation system of the UAV collaborative combat, the following aspects are mainly completed:

At first, the expert PCA method is proposed and is used to complete the construction of the indicator system, which significantly reduces the workload of the indicator selection. Then, during the modeling of the contribution degree, a subjective evaluation model based on the modified gray correlation analysis method and an objective evaluation model based on the minimum entropy weight method are constructed to obtain the subjective and objective weights of the various indicators. Finally, a more reasonable weight allocation coefficient is determined by the minimum relative entropy principle, and the combined subjective and objective efficiency evaluation model is obtained.

Through case analysis, the evaluation indicator system and the contribution model of the UAV collaborative combat effectiveness constructed in this article are reasonable and effective, which provides certain theoretical references for the UAV cooperative combat effectiveness evaluation.

\section{AUTHORS' CONTRIBUTION}

Conceptualization: Sun L, Zhang J; Methodology: Sun L, Zhang J; Investigation: Chang J; Writing - Original Draft: Sun L; Writing - Review and Editing: Zhang J; Funding Acquisition: Fu Z; Resources: Zou J; Supervision: Zou J.

\section{DATA AVAILABILITY STATEMENT}

All the datasets were generated during the current study.

\section{FUNDING}

National Thirteen-Five Equipment Pre-Research Foundation of China

Grant No. 61403120207

Aeronautical Science Foundation of China

Grant No. 20185142003

[https://doi.org/10.13039/501100004750]

National Defense Basic Scientific Research Program of China

Grant No. JCKY2018419C001

Science and Technology Innovative Talents in Universities of Henan Province

Grant No. 21HASTIT030 
Young Backbone Teachers in Universities of Henan Province

Grant No. 2020GGJS073

Leading Talents of Science and Technology Innovation in the Central Plains of China

Grant No. 194200510012

\section{REFERENCE}

Diao X, Fang Y, Wu Y, Fu X (2014) Simulation analysis on air-to-air missile allowable launch envelope about cooperative air combat of multi-fighter formation. Journal of Beijing University of Aeronautics and Astronautics 40(3):370-376. Chinese. https://doi.org/10.13700/j.bh.1001-5965.2013.0237

Fan J, Li D, Li R (2018) Evaluation of MAV/UAV Collaborative Combat Capability Based on Network Structure. Int J Aerosp Eng 2018: 5301752. https://doi.org/10.1155/2018/5301752

Hanson CL, Oliver EJ, Dodd-Reynolds CJ, Pearsons A, Kelly P (2020) A modified Delphi study to gain consensus for a taxonomy to report and classify physical activity referral schemes (PARS). Int J Behav Nutr Phys Act 17:158. https://doi. org/10.1186/s12966-020-01050-2

Jiang J, Wang M, Wang J (2020) Combat Effectiveness Evaluation of Long-range Rocket Launcher under UAV Cooperation. Chinese Journal of Ordnance Equipment Engineering 41(7):202-207. Chinese. https://doi.org/10.11809/bqzbgcxb2020.07.040

Li J-f, Xiang H-c, Yan Y-r, Li Z-x (2020) Combination Weighting Method and Application Based on Maximizing Deviations. Fire Control \& Command Control 45(6):18-23.

Luo C, Chen Y, Wang L, Wang Z, Chang Z (2019) Effectiveness evaluation method of system-of-systems based on operation loop and improved information entropy. Systems Engineering and Electronics 41(1):73-80. Chinese. https://doi.org/10.3969/j. issn.1001-506X.2019.01.11

Qin Y, Zhang X, Gao G, Wang K (2020) Effectiveness Evaluation of Anti-Ship Missile Combat System Based on ANP-Entropy Method. Journal of Ordnance Equipment Engineering 41(5):48-53. Chinese. https://doi.org/10.11809/ bqzbgcxb2020.05.010

Shan B, Chen J, Wang Q (2014) Sensitivity analysis of debris flow based on the theory of minimum entropy and unascertained measure. Rock and Soil Mechanics 35(5):1445-1454. Chinese. https://doi.org/10.16285/j.rsm.2014.05.015

Tian J, Zhang P, Wang Z, Wang Y (2004) The Integrating Model of Expert's Opinion Based on Delphi Method. Systems Engineering - Theory \& Practice 24(1):57-62,69. Chinese. https://doi.org/10.3321/j.issn:1000-6788.2004.01.010

Tong Q, Tong Z-x, Gu X-d, Lu Y-l, Guo H, Chai S-j (2011) Target Threat Assessment for Air Combat Based on Information Entropy. Mathematics in Practice and Theory. 
Wang P, Zhou Y, Wang J, Zhu Cheng, Chen C, Zhang W (2017) A framework for key element evaluation of combat system. 2017 IEEE International Conference on Systems, Man, and Cybernetics (SMC). IEEE; Banff, Alberta, Canada. https://doi. org/10.1109/SMC.2017.8123103

Wang S, Dong R, Xie H, Jia X (2016) Delphi method and its application progress in building index system. Journal of Bengbu Medical College 41(5):695-698. Chinese. https://doi.org/10.13898/j.cnki.issn.1000-2200.2016.05.048

Wang YC, Ao ZG, Gong YX, Jiang YY, Zhao TX (2013) Based on Interval-Valued Intuitionistic Fuzzy Information of Joint Operations Commander and Staff Ability Assessment. Advanced Materials Research, 756-759:4694-4698. Chinese. https:// doi.org/10.4028/www.scientific.net/AMR.756-759.4694

Zhang W-q, Xi Z-l (2021) Application of Delphi method in screening of indexes for measuring soil pollution value evaluation. Environ Sci Pollut Res 28:6561-6571. Chinese. https://doi.org/10.1007/s11356-020-10919-5

Zhang X, Xu R-m (2017) Research on the Joint Operational Synergy Effectiveness Evaluation System Based on Combination Weighting Method. Fire Control \& Command Control 42(7):56-60. Chinese. https://doi.org/10.3969/j. issn.1002-0640.2017.07.013 